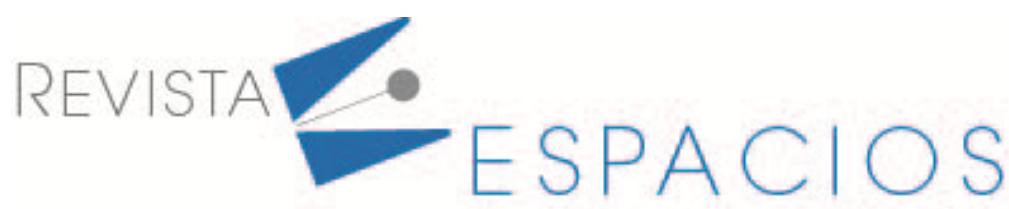

Vol. 42 (24) 2021 • Art. 2 (Especial Publicaciones académicas y COVID-19)

Recibido/Received: 10/12/2021 • Aprobado/Approved: 15/12/2021 • Publicado/Published: 30/12/2021

DOI: $10.48082 /$ espacios-a21v42n24p02

\title{
Tres revistas: Tres visiones
}

\section{Three journals: three approaches}

\begin{abstract}
FERRER-E, Argelia ${ }^{1}$
\section{Resumen}

Se presentan tres entrevistas a los académicos que dirigen las revistas científicas de Comunicación Social en el ámbito iberoamericano Comunicar, Comunicación y Sociedad, y Disertaciones. Sus respuestas a un cuestionario sencillo permiten conocer los retos y pespectivas de esas publicaciones, así como las deficiencias y fortalezas de los artículos que reciben.

Palabras clave: revistas académicas de comunicación, comunicar, comunicación y sociedad, disertaciones

Abstract

Three relevant scholars who perform as editors of three widely read journals on Iberoamerican mass communication, Comunicar, Comunicación y Sociedad, y Disertaciones, are interviewed. Their answers to a set of simple questions allows knowledge of the challenges and perspectives of their publications as well as the strength and weaknesses of the articles received as submisions.

Key words: scholarly journals on communication, comunicar, comunicación y sociedad, disertaciones
\end{abstract}

\section{Introducción}

Quienes se dedican a la investigación, una vez que concluyen un trabajo o tienen avances que presentar, se preguntan en dónde publicar los resultados obtenidos para compartirlos con la comunidad académica. Si el autor es de Iberoamérica tiene múltiples opciones, las cuales han ido creciendo y se han ido abriendo con las posibilidades que brinda la red, pues van desde la llamada "ruta verde" --publicar en un repositorio institucional o temático el artículo, con o sin revisión--; la "ruta dorada", que permite el acceso abierto; o la "ruta híbrida", en la cual el autor paga por publicar en una revista de acceso el libre.

Estos no son los únicos caminos, aunque parecen los más transitados, con sus sugestivos nombres. En Universo Abierto, el blog de la biblioteca de Traducción y Documentación de la Universidad de Salamanca, se explican otros, como la "ruta bronce", referida a artículos de libre lectura en las páginas de los editores, pero sin una licencia abierta explícita que permita su distribución y reutilización; y la "ruta diamante" que intenta cubrir la brecha existente entre los modelos verde y dorado, tomando en cuenta los dos aspectos más críticos de ambos modelos, como son los costes y la calidad, "siendo el único modelo que garantiza la sostenibilidad de la publicación de acceso abierto". (Universo Abierto, 2019).

\footnotetext{
${ }^{1}$ Periodista. Profesora jubilada de la Universidad de Los Andes. Venezuela. Email: argeliaferrer@gmail.com
} 
Las revistas académicas de Comunicación Social en Iberoamérica ofrecen diversas rutas y también presentan diferentes retos, fortalezas y debilidades, así como visiones de su futuro. En este número de Espacios presentamos las opiniones de los responsables de tres de estas publicaciones especializadas: Comunicar, Disertaciones y Comunicación y Sociedad.

Estas tres revistas tienen en común el objetivo de publicar artículos referidos al mundo académico de la Comunicación Social, pero cada una presenta un origen y unas características diferentes, como han explicado los académicos Ignacio Aguaded (editor jefe de Comunicar), Gabriela Gómez Rodríguez (editora en jefe de Comunicación y Sociedad) y Carlos Arcila Calderón (director de Disertaciones).

Las preguntas que les formulamos fueron: ¿Cuál es el principal reto actual de una revista académica en el área de la Comunicación Social?, ¿Cuáles son las deficiencias y fortalezas más comunes en los artículos que reciben? y ¿Cómo vislumbra el futuro de estas publicaciones en general y en la que usted dirige, en particular? Las respuestas se presentan a continuación.

\subsection{Comunicar}

Comunicar es una revista científica trimestral, bilingüe en español e inglés en todos sus artículos, y abstracts en portugués, chino y ruso. Está presente en 805 bases de datos internacionales, plataformas de evaluación de revistas, directorios selectivos, portales especializados y catálogos hemerográficos. Los manuscritos recibidos los someten a un riguroso y transparente sistema ciego de evaluación, con compromisos éticos de transparencia y puntualidad, sistemas antiplagio (CrossCheck), métricas alternativas (PlumX, Dimensions). Asimismo ofrecen un alto nivel de visibilización con múltiples sistemas de búsqueda.

La revista se especializa en educomunicación y publica monográficos especializados en temas de máxima actualidad en formato doble -impreso y online- este último accesible a texto completo de forma gratuita. Editada por Comunicar, asociación profesional no lucrativa, veterana en España (32 años) en educomunicación, que colabora con múltiples centros y universidades internacionales, fundada y presidida por el Dr. Ignacio Aguaded. (Revista Comunicar, 2021). (Imagen 1)

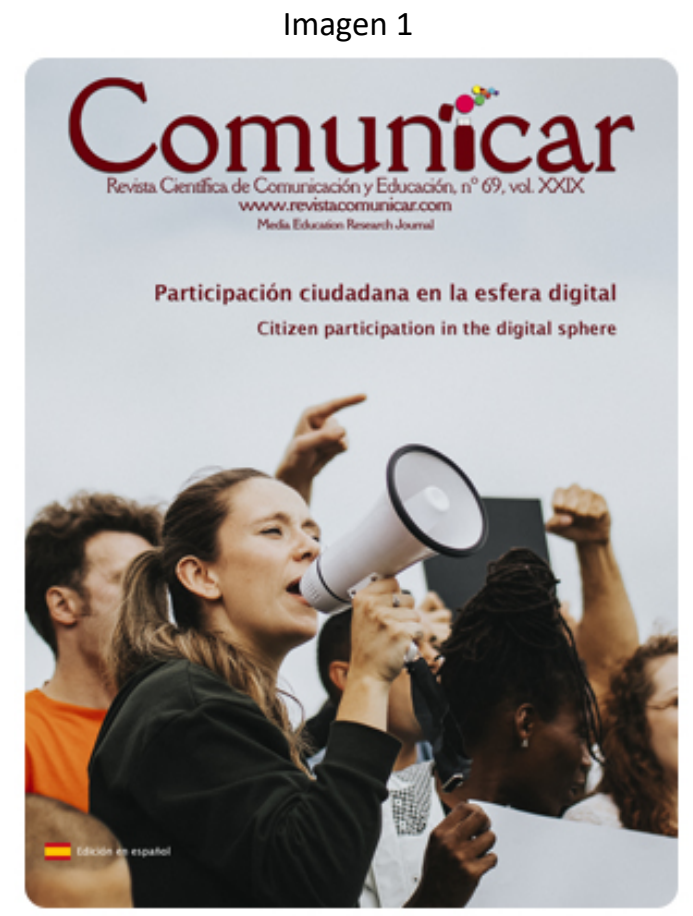

Fuente: Revista Comunicar (2021) 


\section{Entrevista a Ignacio Aguaded, Editor Jefe de la Revista Científica Comunicar}

-Pregunta: ¿Cuál es el principal reto actual de una revista académica en el área de la Comunicación Social?

-Respuesta: Las revistas académicas de acceso abierto se enfrentan principalmente a tres dificultades: cantidad, calidad y sostenimiento económico.

La cantidad, el alto número de artículos que se reciben para ser valorados en los monográficos, tanto en las secciones monográficas como en las misceláneas, supone un trabajo intensivo y exigente a los editores que ha decidir que trabajos cumplen con los parámetros más exigentes para ser publicados: novedad, originalidad, relevancia y transferencia social y científica.

La calidad, las revistas open access que aspiran a la excelencia internacional reciben numerosos artículos que no cumplen con los parámetros de calidad. En cambio, las revistas con embargo, que no son de acceso libre y pertenecen a grandes multinacionales, cuentan con equipos multiprofesionales y tecnologías avanzadas que se ven beneficiadas en los rankings en detrimento de las revistas gratuitas. Además, desgraciadamente el tiempo de revisión y publicación en las revistas open access suele ser dilatado perjudicando a la comunidad científica.

El sostenimiento económico de la revista depende de la financiación y de la aportación de los partners, por lo que el mantenimiento a largo plazo de los múltiples costes (plataformas digitales, procesos editoriales...) puede suponer una gran dificultad en algunos momentos.

-P: ¿Cuáles son las deficiencias y fortalezas más comunes en los artículos que reciben?

-R: Deficiencias:

Incumplimiento de aspectos formales

Artículos fuera del scope de la revista

Escaso rigor metodológico

Envíos duplicados y fuera de plataforma

Fortalezas:

Artículos con muestras comparativas internacionales

Conclusiones de gran impacto

Trabajos novedosos y relevantes, con una fuerte carga de transferencia social.

Autores/as de gran reconocimiento internacional

-P: ¿Cómo vislumbra el futuro de estas publicaciones en general y en la que usted dirige, en particular?

-R: El movimiento open access es imparable. Los investigadores que planifican y ejecutan sus investigaciones no quieren ni deben pagar por consultar los trabajos de otros colegas. Internet se ha convertido en un universo abierto y gratuito en gran parte de sus productos, al menos en una primera instancia. Se estima que el $70 \%$ de las revistas son ya o aspiran a ser de acceso abierto. Las revistas con artículos de las rutas verde, dorada e híbrida están creciendo de forma considerable y van dejando arrinconadas a las que se mantienen con embargos.

Con respecto a Comunicar, la revista vislumbra un futuro en constante crecimiento, tiene 29 años de edición y 1.890 artículos de investigación publicados en open access desde sus inicios, que ofrecen credibilidad y una marca de prestigio creciente, siendo hoy un referente en la comunidad iberoamericana, como revista en español, de máxima indexación internacional (top 1\% mundial) y todo ello en open access desde el inicio, al servicio de la comunidad sin fines lucrativos. 
En indexaciones activas en 2021/22, Comunicar es revista top mundial: $2^{\text {a }}$ del mundo en SCOPUS y 7ạ del mundo en JCR. Con un crecimiento imparable desde 2007 que entra en el Journal Citation Reports, Comunicar desde hace años se mueve en los primeros puestos mundiales, además de liderar los índices latinoamericanos: DIALNET

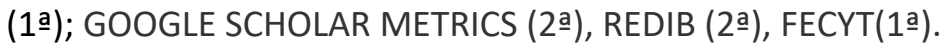

\subsection{Comunicación y Sociedad}

Comunicación y Sociedad es una revista especializada en el área de la comunicación social, dirigida a investigadores y estudiantes dentro de la misma. Su objetivo ser un medio de difusión de avances y resultados de investigación, así como de las teorías y metodologías afines, y debates actuales en el área.

Esta revista de Universidad de Guadalajara (México) -en específico del Departamento de Estudios de la Comunicación Social, Centro Universitario de Ciencias Sociales y Humanidades - publica artículos de alto nivel, producto de investigaciones realizadas sobre temas y debates que forman parte de las preocupaciones en el campo de la comunicación social.

Comenzó a publicarse en 1987 en forma de Cuaderno y fue evolucionando en calidad y periodicidad. En 2015 se convierte en una revista en formato electrónico, bajo el gestor Open Journal Systems y en 2019 cambia de cuatrimestral a ser de publicación continua.

Está registrada en los siguientes directorios y bases de datos: Scopus (Q2), Sistema de Clasificación de Revistas Mexicanas de Ciencia y Tecnología del Conacyt, México; SciELO México, SciELO Citation Index, Latindex-Catálogo, CLASE, REDIB, DIALNET, HAPI. (Comunicación y Sociedad, 2021). (Imagen 2)
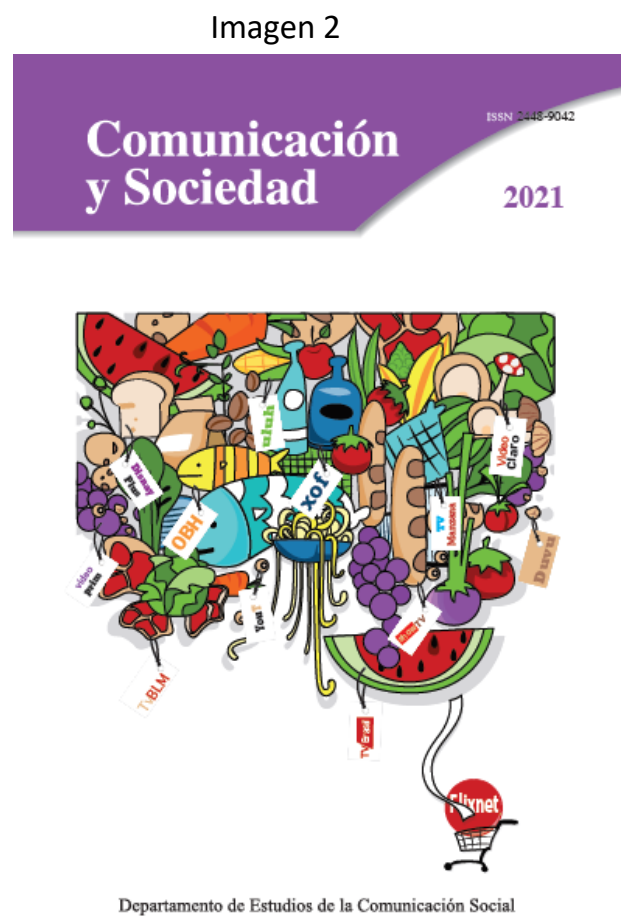

Universidad de Guadalajara

Fuente: Comunicación y Sociedad (2021) 


\section{Entrevista a Gabriela Gómez Rodríguez, Editor en Jefe de Comunicación y Sociedad}

-Pregunta: ¿Cuál es el principal reto actual de una revista académica en el área de la Comunicación Social?

-Respuesta: Son muchos retos, no solo uno. Uno de los principales es conservar las revistas, pues cada vez más hay menores presupuestos. Más aún cuando la edita una universidad pública, lo cual es nuestro caso.

Otro reto es mantener la excelencia en la calidad de los artículos. Tener el poder de convocatoria para recibir artículos originales, con temáticas que aporten al campo de estudios y al pensamiento latinoamericano principalmente.

En caso que una revista esté indizada ya sea en WoS o Scopus, el reto es mantenerse en ese índice y escalar en los sitios, buscando un equilibrio para no perder identidad.

Otro reto (muy importante) es publicar investigaciones originales que respondan a las problemáticas actuales y que generen una discusión y aporte en el campo.

-P: ¿Cuáles son las deficiencias y fortalezas más comunes en los artículos que reciben?

-R: Una de las deficiencias es que las preguntas que se plantean en los estudios siguen una línea de conocimiento particular, a veces hegemónica, sin cuestionar mucho si hay otras más. Considero que deberíamos ser más críticos al momento de construir las preguntas e incluir teorías y metodologías innovadoras y que respondan a los contextos de esta región, y no seguir tanto lo que realizan los investigadores anglófonos principalmente.

Una de las fortalezas es el enfoque principalmente iberoamericano en los estudios, el hecho de incluir también textos con reflexiones teóricas y metodológicas es una de las fortalezas de los artículos para la revista.

-P: ¿Cómo vislumbra el futuro de estas publicaciones en general y en la que usted dirige, en particular?

-R: Vislumbro una revista ubicada en los mejores índices, conocida en todo el mundo (estadísticas que tenemos nos muestran que es leída incluso en Rusia), publicando artículos y autores de primer nivel pero también incluyendo investigadores jóvenes, con temáticas pertinentes y actuales, además, difundiendo el conocimiento producido no solo a través de los artículos, sino haciendo uso de redes sociales, llegando a un público especializado pero también general.

Es un reto mantener una publicación, espero que poco a poco logremos conformar un equipo humano más estable, para poder realizar las labores de diagramación y producción de la revista sin depender tanto de financiamientos. Y poder publicar más artículos al año.

El futuro ya llegó y hay muchos cambios, como publicar continuamente, o publicaciones adelantadas, preprint donde existe la posibilidad de recibir críticas de lectores, todo un proceso más transparente en todos sentidos que enriquezca los textos.

EL futuro dependerá de qué tanto las universidades públicas puedan encontrar financiamiento para sostener las revistas $u$ otras publicaciones, que aunque se publiquen en formato electrónico, es un gasto enorme, si no, tendremos que buscar otra maneras de obtener recursos para poder seguir vigentes.

\subsection{Disertaciones}

El Anuario Electrónico de Estudios en Comunicación Social Disertaciones es una publicación semestral, digital, de acceso abierto e inmediato a su contenido bajo el principio de disponer gratuitamente la investigación al público para incentivar un mayor intercambio de conocimiento global. No se generan cobros a los autores por la gestión, evaluación y publicación de los artículos. Su finalidad es exponer trabajos empíricos de investigación inéditos en 
el área temática de la Comunicación Social, específicamente en las líneas de Sociedad y Tecnologías de la Información y la Comunicación; Comunicación y Política; Periodismo; y Comunicación y Cultura.

Es una publicación arbitrada e indizada de la Universidad del Rosario (Colombia), la Universidad de Los Andes (Departamento de Comunicación Social, Grupo de Investigación Comunicación, Cultura y Sociedad de la ULA Táchira y el Laboratorio de Investigación Educativa Simón Rodríguez - LIESR de la ULA Trujillo) y la Universidad Complutense de Madrid, España (Grupo de Mediación Dialéctica de la Comunicación Social - MDCS). Sus textos se publican en español, inglés y portugués.

Está indizada en: IB-Índice bibliográfico. IBN-Publindex de Colciencias (C). BBCS-Bases bibliográficas con comité de selección: Research Data Bases -EBSCO: Communication Source; Dialnet de la Universidad de la Rioja; RedALyC; DOAJ; REDIB; CIRC (Ciencias sociales-C). Bases de datos bibliográficas:. Google Scholar; Latindex de la UNAM; Revencyt; Rebiun; Red Iberoamericana de Revistas de Comunicación y Cultura; Ulrich's International Periodicals Directory; Scilit; ERIH; Electronic Journals Library (Elektronische Zeitschriftenbibliothek o EZB); Vlex; LatinREV; Ranking Rev-Sapiens. (Anuario Electrónico de Estudios en Comunicación Social Disertaciones, 2019). (Imagen 3).

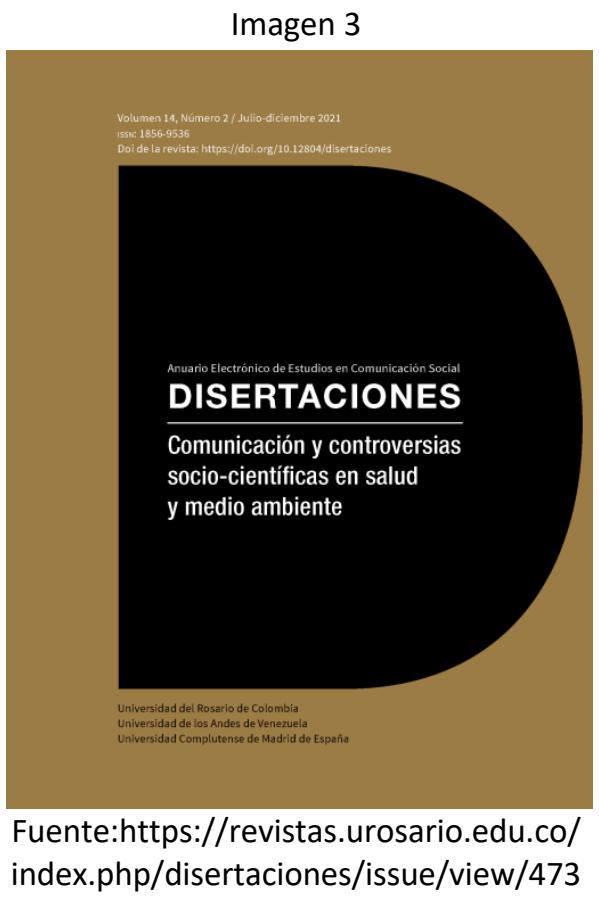

\section{Entrevista a Carlos Arcila Calderón, Director de Disertaciones}

-Pregunta: ¿Cuál es el principal reto actual de una revista académica en el área de la Comunicación Social?

-Respuesta: El principal reto es la difusión de conocimiento científico y académico de calidad. Ha existido una explosión de revistas y de medios dedicados a la difusión de la investigación en comunicación en la última década gracias a Internet, lo cual es bueno para el campo. Sin embargo, la academia Iberoamericana sigue manteniendo la tradición del ensayo y le ha costado incluir resultados de investigación empírica o teórica de calidad. Por ello, una revista (aún) emergente como Disertaciones tiene como reto reforzar la publicación de manuscritos resultados de estudios empíricos de calidad que ayuden a mejorar el trabajo que realizamos los investigadores en la región. Adicionalmente, vista la multiplicidad de temáticas, Disertaciones busca explorar aquellos temas de relevancia científica que han tenido poca cabida en otros medios y que requieren un espacio para su difusión. 
Por esto, un reto adicional es detectar los temas y líneas de investigación que aún no han tenido acogida en las grandes revistas del área para poder dar salida estos artículos.

-P: ¿Cuáles son las deficiencias y fortalezas más comunes en los artículos que reciben?

-R: La principal fortaleza es que suelen ser artículos innovadores, llenos de buenas ideas y del abordaje de temas trascendentes para la comunidad científica en comunicación. La principal deficiencia es que hay muchas carencias metodológicas y de justificación teórica que dificultan la evaluación de los textos que recibimos.

-P: ¿Cómo vislumbra el futuro de estas publicaciones en general y en la que usted dirige, en particular?

-R: La publicaciones científicas en comunicación tenderán a especializarse aún más: en vez de una revista de una universidad o de una asociación científica, tenderán a abordar temas muy particulares. En el caso de Disertaciones, creo que mantendrá los números monográficos como forma de responder a esta hiper especialización en la investigación, pero también mantendrá una sección abierta destinada a los temas emergentes. Adicionalmente, Disertaciones mantendrá una estrategia de "ciencia abierta", que muchas revistas ya tienen: no solo en open access sino intentando llegar al mayor número de investigadores por diferentes canales (como redes sociales) y en diferentes lenguajes (visualizaciones, videos, etc.) y si es posible al público no especializado (usando formatos alternativos de divulgación de los manuscritos originales).

\section{Referencias}

Anuario Electrónico de Estudios en Comunicación Social Disertaciones (2019). Recuperado de https://revistas.urosario.edu.co/index.php/disertaciones/about

Comunicación y Sociedad (2021). Recuperado de http://www.comunicacionysociedad.cucsh.udg.mx/index.php/comsoc/about

Revista Comunicar (2021) Recuperado de https://www.revistacomunicar.com/

Universo Abierto (2019). Las 5 rutas para llegar al acceso abierto: verde, dorada, bronce, híbrida y diamante. Recuperado de https://universoabierto.org/2019/03/13/las-5-rutas-para-llegar-al-accesoabierto-verde-dorada-bronce-hibrida-y-diamante/

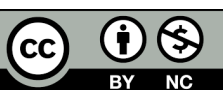

Esta obra está bajo una Licencia Creative Commons Atribución-NoComercial 4.0 Internacional 